CHERCHER, REPÉRER, AVANCER

\title{
LES PROFESSIONS INTELLECTUELLES ENTRE L'ÉTAT, L'ENTREPRENEURIAT ET L'INDUSTRIE
}

\section{Gisèle Sapiro}

\section{La Découverte | «e Mouvement Social »}

2006/1 n ${ }^{0} 214 \mid$ pages 3 à 18

ISSN 0027-2671

Article disponible en ligne à l'adresse :

http://www.cairn.info/revue-le-mouvement-social-2006-1-page-3.htm

\section{Pour citer cet article :}

Gisèle Sapiro, « Les professions intellectuelles entre l'État, l'entrepreneuriat et l'industrie », Le Mouvement Social 2006/1 (nº 214), p. 3-18.

DOI $10.3917 / \mathrm{lms} .214 .0003$

Distribution électronique Cairn.info pour La Découverte.

(C) La Découverte. Tous droits réservés pour tous pays.

La reproduction ou représentation de cet article, notamment par photocopie, n'est autorisée que dans les limites des conditions générales d'utilisation du site ou, le cas échéant, des conditions générales de la licence souscrite par votre établissement. Toute autre reproduction ou représentation, en tout ou partie, sous quelque forme et de quelque manière que ce soit, est interdite sauf accord préalable et écrit de l'éditeur, en dehors des cas prévus par la législation en vigueur en France. Il est précisé que son stockage dans une base de données est également interdit. 


\section{Les professions intellectuelles entre l'Etat, l'entrepreneuriat et l'industrie}

\section{par Gisèle SAPIRO*}

$\mathbf{L}$ 'histoire sociale des professions, qui a connu un important développement depuis deux décennies, notamment en Allemagne et en Italie (1), laisse tradi tionnellement de côté les métiers de la création pour ne se consacrer qu'aux professions intellectuelles considérées comme "utiles": avocats, médecins, enseignants, ingénieurs, architectes. L'histoire des intellectuels, qui s'est centrée sur les écrivains et les artistes plutôt que sur les précédentes professions, a mis de son côté l'accent sur la question de leur engagement politique (2). Ce déséquilibre, qui tient en partie à la division du travail entre histoire sociale des élites (ou du Bildungsbürgertum en Allemagne) et histoire culturelle (3), s'explique aussi par des raisons historiques: alors que les premières sont des professions organisées, dont on peut retracer le processus d'institutionnalisation, les métiers de la création sont des activités fortement personnalisées et individualistes qui constituent un défi pour la sociologie des professions (4). Le degré et le mode d'institutionnalisation ont des incidences sur le type d'action collective. On peut émettre, en effet, l'hypothèse

* Directrice de recherche au CNRS (Centre de sociologie européenne).

(1) G. Cocks and K. JARAusch, German Professions, 1800-1950, New York, Oxford University Press, 1990 ; M. MaLATESTA (ed.), Society and Professions in Italy, 1860-1914, Cambridge, Cambridge University Press, 1995.

(2) Parmi les nombreuses publications consacrées à ce thème, on citera notamment les travaux de P. ORY et J.-F. SIRINELLI, Les Intellectuels en France, de l'Affaire Dreyfus à nos jours, Paris, Armand Colin, $2^{e}$ éd., 1992 ; C. ProchASSON, Les Intellectuels, le socialisme et la guerre (1900-1938), Paris, Seuil, 1993 et, avec A. RASMUSSEN, Au nom de la patrie. Les intellectuels et la Première Guerre mondiale (1910-1919), Paris, La Découverte, 1996 ; et sur la relation entre engagement et obéissance politique F. MATONTI, Intellectuels communistes. Essai sur l'obéissance politique. La Nouvelle Critique (1967-1980), Paris, La Découverte, 2005.

(3) Cf. C. CHARLE, "Intellectuels, Bildungsbürgertum et professions au XIXe siècle. Essai de bilan historiographique comparé (France, Allemagne) ", Actes de la recherche en sciences sociales, n 106-107, mars 1995, p. 85-95. Voir, par exemple, W. ConzE et J. KoCKA (Hg.), Bildungsbürgertum im 19. Jahrhundert, vol. I, Bildungssystem und Professionnalisierung in internationalen Vergleichen, Stuttgart, Klett Verlag, 1985.

(4) E. FREIDSON, "Les professions artistiques comme défi à l'analyse sociologique", Revue française de sociologie, XXVII-3, juillet-septembre 1986, p. 431-444.

Le Mouvement Social, n 214, janvier-mars 2006, @ Les Éditions de l'Atelier/Éditions Ouvrières 
que le fort degré de politisation des activités de création (littéraire, en particulier) est lié à leur faible degré de professionnalisation (5). Inversement, la mobilisation pour la reconnaissance de la profession a sans doute contribué à la formation d'un "esprit de corps " qui masque les divisions politiques et les engagements individuels dans les professions traditionnelles (6).

Dès 1985, Madeleine Rebérioux avait pourtant suggéré l'intérêt de mettre en chantier une histoire sociale des intellectuels qui prenne en compte leur position dans l'espace social, l'évolution du statut de leur activité et leurs formes d'organisation et d'association (7). Une telle approche existait pour le XVIII' siècle, avec les travaux de Roger Chartier et Daniel Roche en particulier (8). Elle a été développée pour le XIXe siècle notamment par Christophe Charle, qui a mis au jour la position des différentes fractions intellectuelles dans l'espace social et a proposé une approche comparative au niveau européen (9). Si le sociologue américain Andrew Abbott a montré le profit que l'on peut tirer d'une analyse de la compétition entre professions autour de la "division du travail d'expertise " et des domaines de compétence (jurisdiction), Fritz Ringer, dans son étude comparée des systèmes d'enseignement en France et en Allemagne, souligne de son côté la nécessité de prendre en considération la concurrence entre la culture littéraire humaniste, qui a façonné l'habitus des élites cultivées du XVIII siècle à la fin du XIXe siècle, et la culture scientifique montante, caractérisée par la spécialisation, comme principes de légitimation du pouvoir symbolique (10). C'est dans cette perspective que s'inscrit le présent numéro. Il aborde

(5) G. SAPIRO, "Forms of politicization in the French literary field ", Theory and Society, 32, 2003, p. 633-652.

(6) Voir P. BOURDIEU, "Effet de champ et effet de corps", Actes de la recherche en sciences sociales, $n^{\circ}$ 59, septembre 1985, p. 73 ; J. D. EluIs, The Physicians-Legislators of France. Medicine and Politics in the Early Third Republic, 1870-1914, Cambridge, Cambridge University Press, 1990. Voir aussi le livre récent de L. ISRAËL, Robes noires, années sombres. Avocats et magistrats en résistance pendant la Seconde Guerre mondiale, Paris, Fayard, 2005.

(7) M. REBÉRIOUX, "Les intellectuels français au XXe siècle : quelques questions ", in B. KÖPECZI et J. LE GoFF (dir.), Intellectuels français, intellectuels hongrois - XIII'-XX' siècle, Budapest-Paris, Akadémiai Kiadó-CNRS, 1985.

(8) R. CHARTIER, "Espace social et imaginaire social. Les intellectuels frustrés au XVI" siècle", Annales ESC, n 3, mai-juin 1982, p. 389-400 ; D. RocHE, "L'intellectuel au travail ", ibid., p. 465-480.

(9) Voir en particulier C. CHARLE, Naissance des "intellectuels" 1880-1900, Paris, Minuit, 1990 ; La République des universitaires 1870-1940, Paris, Seuil, 1994 et Les Intellectuels en Europe au XIX siècle. Essai d'histoire comparée, Paris, Seuil, 1996. Voir aussi, dans la même perspective, J.-L. FaBIANI, Les Philosophes de la République, Paris, Minuit, 1985. Pour une réflexion sur la démarche comparatiste, voir également M. TREBITSCH et M.-C. GRANJON (dir.), Pour une histoire comparée des intellectuels, Bruxelles, Complexe, 1998.

(10) A. ABвоTT, The System of Professions. An Essay on the Division of Expert Labor, Chicago, The University of Chicago Press, 1988. F. RINGER, Fields of Knowledge. French Academic Culture in Comparative Perspective 1890-1920, Cambridge-New York-Paris, Cambridge University Press - Éd. de la MSH, 1992. Il n'est pas possible de recenser ici tous les travaux existants sur les systèmes d'enseignement, qui sont centraux pour comprendre l'orientation des carrières et les rapports entre titre et poste. Citons ici uniquement, pour la France, A. Prost, Histoire de l'enseignement en France, 1800-1967, Paris, Armand Colin, 1968 ; G. WeIsz, The Emergence of Modern Universities in France (1863-1914), Princeton, Princeton University Press, 1983 ; J. VERGER (dir.), Histoire des universités en France, Toulouse, Privat, 1986 (en particulier les contributions de V. Karady). 
le problème de l'organisation professionnelle d'activités moins institutionnalisées, et donc moins étudiées sous cet angle, en s'intéressant également aux tentatives de professionnalisation qui ont échoué, questions dont les débats récents suscités par l'instauration du prêt payant des livres en bibliothèque ou par le statut des intermittents du spectacle ont rappelé l'actualité.

Par son caractère téléologique, par sa supposition d'un modèle de développement unique, la notion de "professionnalisation " forgée par la sociologie américaine se révèle toutefois réductrice dès qu'on historicise les conditions de codification de ces activités, les tentatives - réussies ou ratées - d'unification du corps professionnel et les processus d'homogénéisation, voire de fermeture du corps (11). Au terme de "professionnalisation " Andrew Abbott a proposé de substituer celui de "développement professionnel " pour éviter l'implication téléologique du premier (12). Si donc on emploiera parfois ici le terme commode de "professionnalisation ", c'est pour désigner, en le resituant dans son contexte historique, le phénomène concret de développement de l'organisation professionnelle de nombre d'activités intellectuelles. L'étude historique des formes d'organisation des professions intellectuelles et de leurs instances constitue, de ce point de vue, une contribution majeure à une meilleure compréhension de leur mode d'institutionnalisation, entre l'État, l'entrepreneuriat et l'industrie, après le démantèlement des structures corporatistes d'Ancien Régime.

\section{Les revendications d'autonomie et de reconnaissance du travail intellectuel}

Les conditions d'exercice des métiers intellectuels varient tout d'abord selon le type de statut qui leur est associé. On peut en distinguer trois principaux : indépendant, fonctionnaire et employé. Au XIX siècle, le modèle bourgeois de l'indépendance et de l'entrepreneuriat prévalait dans les sociétés de tradition libérale comme l'Angleterre et les États-Unis. Dans les États bureaucratiques et autoritaires, comme les empires français, allemand et habsbourgeois, une partie des services intellectuels, tel l'enseignement, étaient assimilés à la fonction publique, ce qui permettait de les libérer des contraintes du marché et d'empêcher que les intérêts particuliers, économiques notamment, ne l'emportent sur l'intérêt général, tout en les contrôlant sur le

(11) Voir les critiques de cette notion par M. MAURICE, "Propos sur la sociologie des professions", Sociologie du travail, XIV-2, avril-juin 1972, p. 213-225 ; J.-M. CHAPOULIE, "Sur l'analyse sociologique des groupes professionnels ", Revue française de sociologie, XIV-1, janvier-mars 1973, p. 86-114; J. HELlBRON, "La "professionnalisation" comme concept sociologique et comme stratégie des sociologues ", in Historiens et sociologues aujourd'hui, Journées d'études annuelles de la Société française de sociologie, Université de Lille I, $14-15$ juin 1984. Voir également C. DuBAR et P. TRIPIER, Sociologie des professions, Paris, Armand Colin, 1998.

(12) A. Аввотт, The System of Professions, op. cit. 
plan idéologique (13). Si ce modèle avait l'avantage de garantir aux professionnels un monopole, il les subordonnait à la hiérarchie bureaucratique. Inversement, la libéralisation totale du marché les livrait aux aléas de la demande et à une concurrence acharnée qui ouvrait la porte au charlatanisme et aux pratiques mercenaires, comme dans le cas de la médecine en France dans la phase radicale de la Révolution française ou dans celui de la presse au XIXe siècle. L'industrialisation et le processus de rationalisation de la production ont conduit à la croissance d'une catégorie d'intellectuels employés par des grandes firmes, ingénieurs, chimistes, etc.

C'est sur le caractère intellectuel de leur activité et sur sa dimension morale que ces groupes professionnels ont fondé leurs revendications sociales, qu'il s'agisse de l'autonomie pour les fonctionnaires et les employés ou de la reconnaissance par l'État et de la réglementation du statut pour les professions libérales et également les employés (14). La distinction entre travail intellectuel et travail manuel s'inscrit dans une longue tradition. Avec le développement des universités à partir du XIII siècle, les arts libéraux se sont progressivement démarqués des arts mécaniques sur cette base (15). Redécouverte au XVII siècle, l'antique distinction du droit romain entre operae liberales et operae illiberales interdisait que les premières soient soumises au contrat de louage, distinguant l'honorarium, manifestation de la reconnaissance du client pour un service inappréciable pécuniairement, de la merces, réservée au travail manuel. Contre l'esprit de lucre et la recherche de bénéfices caractérisant les activités commerciales, elles affichent un désintéressement qui renvoie moins à la gratuité du service qu'à l'idée que le professionnel doit faire passer ses intérêts propres après ceux qui lui sont confiés. En cela, il est proche des fonctionnaires qui doivent subordonner leurs intérêts particuliers à l'intérêt général. La dimension intellectuelle de leur activité et la responsabilité propre aux professionnels fondent également leur revendication d'autonomie, qui implique un statut de fonctionnaire dérogatoire au statut général comme c'est le cas des enseignants du supérieur en Allemagne et en France. Le modèle des officiers ministériels, qui existe en France pour les notaires et les commissaires-priseurs, combine autonomie relative et contrôle de l'État (16). La profession d'avocat est celle qui a développé le plus précocement, et ce dès le XVIII siècle, une idéologie professionnelle fondée entre autres sur les notions de désintéressement et de responsabilité. Comme l'explique Lucien Karpik, "le désintéressement définit une stratégie qui, refusant aussi bien la subordination à l'État que la soumission au marché, entend se lier au public " (17). Ce modèle élaboré

(13) H. SIEgRIST, "The Professions in Nineteenth-Century Europe", in H. KaELBLE (ed.), The European way, New York, Berghahn Books, 2004, p. 68-88.

(14) J. SAVATIER, Etude juridique de la profession libérale, Paris, Librairie générale de droit et de jurisprudence, 1947, section II et III.

(15) J. Le GofF, Les Intellectuels au Moyen Âge, Paris, Seuil, 1957, rééd. 1985. Voir aussi D. Roche, "L'intellectuel au travail", art. cit.

(16) Cf. A. QuEmIN, Les Commissaires-priseurs. La mutation d'une profession, Paris, Anthropos, 1997. Cette profession issue de l'Ancien Régime est restée très corporatiste au sens de marché fermé, de régulation de la concurrence et de contrôle de la reproduction du corps (de père en fils le plus souvent).

(17) L. KARPIK, Les Avocats. Entre l'État, le public et le marché, XIII-XX'e siècle, Paris, Gallimard, 1995, p. 90-91. 
par les avocats anglais et français est revendiqué par les spécialistes d'autres disciplines dont l'activité professionnelle n'est pas réglementée au cours du processus d'institutionnalisation et de légitimation du savoir expert au XIXe siècle.

Le mouvement de professionnalisation prend son essor en Europe à partir des années 1870 , corrélativement aux processus de généralisation de l'éducation, d'industrialisation, d'expansion du secteur tertiaire et de consolidation des identités nationales : il a donné lieu à des analyses d'ensemble dans les cas de la GrandeBretagne, l'Allemagne et l'Italie (18). En France, où les corporations avaient été supprimées par la Révolution en tant que monopoles (la loi Le Chapelier de 1791 (19)), il se développe avec la législation républicaine qui libéralise le droit à l'association. Bien étudié pour les activités intellectuelles les mieux organisées, en particulier les professions juridiques, les médecins, les enseignants, les ingénieurs, les journalistes (20), il l'a peu été pour les moins professionnalisées et, surtout, a rarement fait l'objet d'une approche comparative (21). Les articles réunis ici entendent combler cette lacune en étudiant, selon des perspectives et des échelles différentes, les tentatives plus ou moins réussies de différentes professions intellectuelles pour s'organiser. A côté de l'exemple, mieux connu, des médecins, sur lesquels est cependant apportée une perspective neuve, sont abordées des activités moins professionnalisées et - sans doute de ce fait - moins étudiées sous cet aspect, comme les architectes, les ingénieurs-conseils, les critiques littéraires, les écrivains. Ces

(18) C'est le cas en Grande-Bretagne, en Allemagne et en Italie, où les professions libérales ont contribué à l'unification de l'État ; cf. H. PERKIN, The Rise of Professional Society. England since 1880, Londres-New York, Routledge, 1989; G. Cocks and K. JaRAUSCH, German Professions, op. cit.; C.E. MCClelland, The German Experience of Professionalization. Modern Learned Professions and their Organizations from the Early Nineteenth Century to the Hitler Era, Cambridge, Cambridge University Press, 1991 ; M. MALATESTA (ed.), Society and Professions in Italy, op. cit. En GrandeBretagne, où les effectifs de diplômés de sciences se multiplient par quatre entre 1902 et 1914, de nouveaux types de groupements associatifs et syndicats voient le jour dans les milieux scientifiques, qui se démarquent des sociétés savantes par la prise en considération des conditions matérielles d'exercice des métiers. L'expérience de la coopération entre spécialistes et de la solidarité durant la Première Guerre mondiale donne à ce mouvement une nouvelle impulsion; cf. R. and K. MACLEOD, "The Contradictions of Professionalism : Scientists, Trade Unionism and the First World War ", Social Studies of Sciences, vol. $9, n^{\circ} 1,1979$, p. 1-33. Notons en outre que l'internationalisation des enjeux politiques durant le conflit a favorisé la diffusion des modèles d'organisation d'un pays à l'autre.

(19) Voir S. L. Kaplan, La Fin des corporations, Paris, Fayard, 2001.

(20) A. GRelon (dir.), Les Ingénieurs de la crise. Titre et profession entre les deux guerres, Paris, Éditions de l'EHESS, 1986 ; E. N. SULEIMAN, Les Notaires. Les pouvoirs d'une corporation, Paris, Seuil, 1987 ; L. KARPIK, Les Avocats, op. cit. ; C. CHARLE, "La bourgeoisie de robe en France au XIXe siècle", Le Mouvement Social, n 181, octobre-décembre 1998, p. 53-72 ; J.-P. BARRIĖRE, "Notaires des villes et notaires des champs : les origines sociales d'une "profession" au XIXe siècle", ibid., p. 73-104 ; C. DELPORTE, Les Journalistes en France (1880-1950). Naissance et construction d'une profession, Paris, Seuil, 1998; A. BANCAUD, Une exception ordinaire. La magistrature en France, Paris, Gallimard, 2002 ; G. Le BÉGUEC, La République des avocats, Paris, Armand Colin, 2003 ; M. Offerlé (dir.), La profession politique XIXe-XXe siècles, Paris, Belin, 1999.

(21) Une telle démarche a été entreprise pour les XVIII et XIXe siècles par G. L. GeISON, Professions and the French State, 1700-1900, Philadelphie, University of Pennsylvania Press, 1984. Voir aussi P. Guillaume, "L'émergence des professions intellectuelles", in Id. (dir.), Regards sur les classes moyennes. XIX'-XX'e siècles, Pessac, Éditions de la Maison des sciences de l'homme d'Aquitaine, 1995, p. 117-127. 
articles mettent tous en évidence le rôle de l'État dans l'organisation des professions intellectuelles et artistiques. Ce rôle de l'État est-il une spécificité française? De fait, minoré par la sociologie des professions anglo-saxonne, l'État joue, comme l'ont montré les travaux historiques, un rôle central dans d'autres pays, mais il n'est pas l'unique recours. En témoigne la tentative d'instaurer une participation des auteurs et des lecteurs aux décisions au sein des maisons d'édition dans l'Allemagne fédérale des années 1960, alors même que le projet de loi sur la cogestion dans l'entreprise fait l'objet d'un blocage, tentative dont l'analyse apporte dans ce numéro un contrepoint qui fait ressortir, par contraste, cette spécificité française.

\section{Division du travail intellectuel et conditions inégales de la concurrence}

La spécificité du cas français réside dans la conjonction de plusieurs facteurs: l'héritage de l'organisation corporatiste de l'Ancien Régime, la centralisation étatique et la constitution d'une "noblesse d'État" (22) qui, associés à l'absence de liberté syndicale et associative jusqu'à la fin du siècle, déterminent les conditions inégales de la concurrence entre les groupes professionnels - et en leur sein - au cours du processus de division du travail d'expertise. Ainsi, bien que les médecins, étudiés ici par Robert Nye, aient obtenu du régime impérial un monopole de droit sur la profession par le contrôle de la formation et le permis d'exercer, qui vise à écarter les charlatans et les guérisseurs, la création du corps des officiers de santé maintient une concurrence qui ne sera résolue (au profit des premiers) qu'en 1892, tandis que se multiplient les jeunes diplômés en quête de clients. Les conditions inégales de la concurrence se ressentent de la façon la plus intense dans les professions fortement dépendantes de la commande étatique ou industrielle. C'est le cas aussi bien des architectes, abordés par Denyse Rodriguez-Tomé, que des ingénieurs civils, analysés par Odile Henry. Or, en France, ces deux professions ont dû définir leur identité face aux ingénieurs issus des grands corps de l'État, qui détenaient un quasi-monopole de la commande étatique et qui étendirent leur prérogative au secteur privé dans la deuxième moitié du XIXe siècle. Chez les architectes, les tentatives d'organisation du corps professionnel et d'imposition du diplôme se heurtent à la forte hétérogénéité des conditions d'exercice du métier, notamment entre Paris et la province, mais la profession se structure autour d'une élite professionnelle produite par l'École des Beaux-Arts et autour de l'identité d'artiste affirmée par le code Guadet. Également divisés par des conditions d'exercice du métier très hétérogènes, les ingénieurs-conseils tentent de leur côté d'affirmer le caractère scientifique d'une compétence qui n'est pas certifiée.

(22) P. Bourdieu, La Noblesse d'État, Paris, Minuit, 1989. 
La concurrence est, de même, très prégnante parmi les producteurs culturels dans les secteurs industrialisés comme la presse et l'édition, devenus dépendants de la demande. En effet, si la formation d'un marché des biens culturels a permis dans un premier temps à ceux-ci de s'affranchir du mécénat, elle a entraîné des pratiques de contrefaçon ainsi que des formes de standardisation et de mercantilisation de ces produits. Le milieu professionnel fait appel à l'État pour protéger ces biens, au nom de leur spécificité en tant qu'objets de patrimonialisation, et pour contrebalancer la logique marchande, selon une argumentation brandie encore récemment sous la formule de "l'exception culturelle" lors des débats autour de l'application des accords du GATS (General Agreement on Trade in Services) au cinéma et à l'audiovisuel. Pionnière dans la codification du droit d'auteur, en particulier de son droit moral, et ayant œuuvé à sa reconnaissance au niveau international, la France se trouve cependant, dans la première moitié du XXe siècle, en retard par rapport à d'autres nations où le droit d'auteur fait l'objet d'une législation d'ensemble plus adaptée aux nouveaux supports. Des projets concurrents sont élaborés dans les années 1930, mais la loi tant attendue ne verra le jour qu'en 1957. Ces projets révèlent les tensions, présentes dès la législation révolutionnaire, entre différentes conceptions de l'auteur, propriétaire, travailleur intellectuel ou assumant une mission de service public, qu'analysent Gisèle Sapiro et Boris Gobille. Parallèlement à ces tentatives de codification d'un statut d'auteur, les aléas de la mise en place d'une Caisse des lettres, ancêtre du Centre national du livre, et les débats autour du domaine public payant après la guerre, illustrent les problèmes posés par la solidarité et l'obtention de droits sociaux dans ces milieux fortement individualistes, et où le soutien de l'État est nécessaire pour contrebalancer les conséquences de la logique marchande. Si la tentative, étudiée par Ingrid GilcherHoltey, d'instaurer une participation des auteurs et des responsables éditoriaux aux décisions au sein des maisons d'édition allemandes offre un modèle alternatif qui se dispense du recours à l'État, dans les deux pays la logique capitalistique continue de prédominer, voire de s'intensifier, dans le monde éditorial, où elle est présente dès le XIXe siècle (23).

La surproduction de livres et les méthodes publicitaires de plus en plus agressives de l'édition ont engendré une situation de crise qui touche non seulement les producteurs mais aussi les intermédiaires, en l'occurrence les critiques littéraires. La fondation de l'Association syndicale professionnelle de la critique littéraire en 1902 et ses tentatives de rassembler le milieu de la critique, qu'analyse Marie Carbonnel, témoignent à la fois de la précarisation des conditions d'exercice de cette activité et de la nécessité de la revaloriser. Divisée entre le pôle universitaire, le pôle journalistique et celui des créateurs, elle rencontre des difficultés comparables à celles des écrivains.

Enfin, si le thème de la "surproduction de diplômés" remonte à l'Ancien Régime, il renvoie, à partir du XIXe siècle, à la concurrence accrue dans toutes les professions, qui s'accentue sous la III ${ }^{e}$ République avec le développement de l'Université républicaine et la croissance des effectifs estudiantins. Face aux aléas du statut

(23) Voir J.-Y. MoLLIER, L'Argent et les lettres. Histoire du capitalisme d'édition (1880-1920), Paris, Fayard, 1988. 
d'indépendant ou à la précarité de l'emploi, une tentative, étudiée ici par Alain Chatriot, de constitution de caisses de chômage intellectuel voit le jour dans les années 1930, à l'initiative d'une nouvelle organisation, la Confédération des travailleurs intellectuels (CTI).

\section{Organisation professionnelle et formes d'association}

Les tentatives d'organisation professionnelle qui se multiplient sous la III ${ }^{e}$ République sont le fruit de la nécessité de faire face à ces conditions de la production intellectuelle. Le mouvement de professionnalisation fut largement favorisé en France par le cadre législatif républicain : la loi du 21 mars 1884 autorisant les syndicats professionnels, celle de 1898 sur les associations de secours mutuel, puis celle du

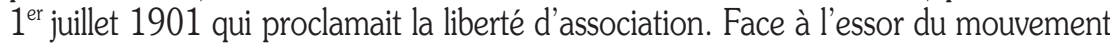
ouvrier, au développement du secteur tertiaire, à la croissance des effectifs universitaires et à la concurrence accrue, les groupes professionnels se posèrent à leur tour la question de leur organisation. L'organisation d'un corps professionnel demande la prise en charge, par une ou plusieurs instances, de fonctions distinctes : la formation, le contrôle de l'entrée dans la profession et de ses conditions d'exercice, l'élaboration d'un code de déontologie et la discipline intérieure, l'entraide, la représentation et la défense des intérêts de la profession.

Pour les professions intellectuelles, dont l'activité se caractérise par l'abstraction (24), la formation, gage de la compétence et donc de la validité de l'expertise, est généralement assurée par l'Université ou par des écoles spécialisées. Dans certaines professions, telles que les architectes et les ingénieurs-conseils, elle n'est cependant pas une condition obligatoire pour exercer le métier, quand elle ne fait pas défaut comme c'est le cas chez les journalistes de la première moitié du siècle ou les écrivains et les critiques littéraires jusqu'à ce jour. Les autres fonctions sont prises en charge par divers types d'instances: chambres, ordres, sociétés, associations, syndicats, caisses de secours. Les conditions d'entrée et la déontologie sont plus ou moins contrôlées selon qu'une ou plusieurs instances représentatives parviennent à se faire reconnaitre par l'État, soit en obtenant le monopole du titre ou de la délivrance d'une carte, par exemple, comme ce sera le cas pour les ingénieurs et pour les journalistes en France dans les années 1930, soit en étant statutairement habilitées à réguler l'accès comme la discipline intérieure de la profession (ordre), selon le modèle des avocats. La fonction d'entraide est assurée par des associations et mutuelles, tandis que la défense des intérêts de la profession dans son ensemble (salaires ou honoraires, conventions collectives, etc.) est prise en charge par les syndicats. Les formes d'organisation varient selon l'histoire nationale et la nature du

(24) A. Аввотт, The System of Professions, op. cit. 
régime, son fondement démocratique ou non, son degré de libéralisme ou d'autoritarisme, son caractère bureaucratique, etc.

Concernant l'accès à la profession et la discipline intérieure, le modèle libéral des avocats s'offrait, on l'a vu, aux autres professions. En l'absence d'un ordre, les groupements associatifs ou les syndicats pouvaient, au-delà des fonctions de sociabilité et d'entraide, jouer un rôle dans la réglementation de l'accès à la profession et l'élaboration d'une identité et une éthique professionnelles. Même lorsqu'elle n'était pas consignée dans un code de déontologie ayant valeur de loi et assorti de sanctions, il pouvait bel et bien exister, dans la pratique et surtout dans l'image que la profession donnait d'elle-même, comme l'explique ici Robert Nye à propos des médecins, un ensemble de représentations formant une éthique professionnelle, sur laquelle la majorité de ses membres s'accordaient et que ces organisations tentaient de formaliser et d'imposer. Sous couvert de moralisation de la profession, ces instances visaient à la fois à réguler la concurrence interne et à asseoir son autorité dans un domaine de compétence dont elle revendiquait l'exclusivité par l'élaboration d'un statut réglementé par l'État, ce que les journalistes et les ingénieurs obtinrent dans les années 1930, alors que la tentative des ingénieurs-conseils de fonder un ordre échoua à trois reprises, comme le montre Odile Henry.

Cette autorité se réclamait, on l'a dit, du caractère "intellectuel " de l'activité, qui permettait à ces professions de se démarquer à la fois du travail manuel et des professions mercantiles. Dimension intellectuelle qui fondait à son tour une éthique de la responsabilité que ces sociétés s'employèrent à codifier. Société de secours mutuel, l'Association syndicale professionnelle de la critique littéraire, qu'étudie Marie Carbonnel, entend ainsi marquer la distinction entre critique et réclame à un moment où le capitalisme d'édition développe des méthodes publicitaires et des moyens de promotion de plus en plus agressifs. De même, comme le montre Denyse RodriguezTomé, la revendication d'une identité d'artiste permet aux architectes de se démarquer de l'activité plus " artisanale " des entrepreneurs. Mais cette identité d'artiste a une autre fonction : elle fonde les revendications de propriété intellectuelle des architectes, à une époque où la propriété industrielle et intellectuelle fait l'objet de conventions internationales (en 1883 et 1886 respectivement). Le rôle majeur joué par la Société des auteurs dramatiques et la Société des gens de lettres depuis leur fondation respectivement en 1791 et en 1838 dans le combat pour l'application de la propriété littéraire à d'autres supports que le livre, en l'occurrence le théâtre et la presse, ainsi que pour la prolongation de sa durée et son adoption au niveau international, illustre cette fonction de défense de la profession.

En l'absence d'ordre, les syndicats médicaux jouèrent, avec l'Association générale de prévoyance et de secours mutuels des médecins de France (AGMF), un rôle dans l'organisation de la profession : des "conseils de famille " furent mis en place à l'échelon départemental et national, qui formaient des juridictions disciplinaires de la profession (25); les médecins syndiqués s'engageaient en outre à observer une tarification d'honoraires minimale.

(25) J. SAVATIER, Étude juridique..., op. cit., p. 101. 
Cependant la liberté syndicale, d'abord réservée aux employés, n'alla pas de soi pour les professions libérales. Alléguant leur parenté avec la fonction publique, certains y voyaient le risque que la défense des intérêts des professionnels soit mise en avant au détriment des clients, dont les intérêts ne peuvent être garantis que par un ordre doté de pouvoirs disciplinaires (26). Dans la profession médicale, qui n'était pas encore unifiée, l'AGMF, fondée en 1858, jouait le rôle de syndicat médical officieux. En 1884, une Union générale des syndicats médicaux de France fut créée de manière illégale. La loi du 30 novembre 1892 réglementa les conditions d'exercice de la profession en imposant le doctorat, abolissant ainsi la concurrence des officiers de santé et homogénéisant la profession (27). Cette même loi autorisa les syndicats médicaux.

Pour les fonctionnaires, la liberté de se syndiquer ne fut acquise que plus tardivement, après avoir fait l'objet de vives discussions (28). Le mouvement fut porté notamment par les instituteurs, tandis que les enseignants du secondaire et du supérieur, organisés en amicales et en associations par catégories et spécialités, lui restèrent plutôt hostiles jusqu'au milieu des années 1920 (29). Cette hostilité à la syndicalisation touche toutes les élites des professions intellectuelles, qui y voient une marque de "prolétarisation" de leur activité, la tendance syndicaliste étant souvent portée par les nouvelles générations pour lesquelles les conditions d'exercice du métier sont plus précaires et qui y trouvent un mode de socialisation dans le milieu professionnel. Recoupant en partie les clivages traditionnels, d'ordre générationnel, géographique (Paris-province), scolaire, socio-économique, qui divisent plus ou moins les professions libérales et artistiques, le clivage entre une élite professionnelle qui forme la bourgeoisie intellectuelle et ce qu'on appelle à l'époque le " prolétariat intellectuel " les traverse toutes de manière plus ou moins exacerbée, des plus organisées (les avocats) aux plus individualistes et vocationnelles (les écrivains). Le projet de caisses de chômage des intellectuels élaboré à l'initiative de la CTI dans les années 1930 illustre la tentative de pallier les aléas des carrières liés aux incertitudes de la demande pour les indépendants et les employés, mais elle se heurte, comme le montre Alain Chatriot, à d'autres formes organisationnelles, notamment celles des classes moyennes, que préfèrent les médecins par exemple (30).

Au lendemain de la Première Guerre mondiale, la liberté syndicale s'étend à toutes les professions en vertu de la loi du 12 mars 1920. Alors que l'Ordre des

(26) Ibid., p. 12.

(27) Cf. J. LÉONARD, La Médecine entre savoir et pouvoir, Paris, Aubier-Montaigne, 1981, et M. Arliaud, Les Médecins, Paris, La Découverte, 1987, p. 5-19.

(28) Voir J. SiwEK-POUYDESSEAU, Le Syndicalisme des fonctionnaires jusqu'à la guerre froide, Lille, Presses universitaires de Lille, 1989; J. WishnIA, The Proletarianizing of the Fonctionnaires: Civil Service Workers and the Labor Movement under the Third Republic, Baton Rouge, Louisiana State University Press, 1990.

(29) Voir P. GERBOD, "Associations et syndicalismes universitaires de 1828 à 1928 (dans l'enseignement secondaire public) ", Le Mouvement Social, n 55 , avril-juin 1966, p. 3-45.

(30) A. CHATRIOT, La Notion de "chômeur intellectuel " et l'action de la CTI (Confédération des travailleurs intellectuels) en France pendant les années 1930, mémoire de maitrise, sous la direction de C. Charle et R. Sanson, Université de Paris I, 1996. Voir aussi L. BolTANSKI, Les Cadres. La Formation d'un groupe social, Paris, Minuit, 1982. 
avocats obtient la reconnaissance officielle de ses attributions par l'État ainsi que la réglementation du titre d'avocat, naissent l'Association nationale des avocats (ANA) et l'Union des jeunes avocats (UJA) (31). Dans les autres professions, la libéralisation du droit à l'association et le développement des sociétés mutuellistes, associés à la spécialisation croissante, ont entrainé la multiplication d'organisations locales, par branches ou spécialités. On assiste alors à un mouvement de regroupement de ces initiatives éparses sur la base des intérêts de la profession dans son ensemble. La Confédération des syndicats médicaux français voit le jour en 1928 et réclame dès l'année suivante la création d'un ordre des médecins, projet mené par des médecins proches de l'Action française qui n'aboutira qu'en 1940 (32). Prenant exemple sur les plus anciennes, avocats et médecins, les professions nouvelles, journalistes et ingénieurs, cherchent aussi à s'organiser. Créé en 1918 pour défendre les intérêts moraux et matériels des journalistes, dont les conditions de travail se sont dégradées, le Syndicat des journalistes s'arroge une fonction de moralisation analogue à celle du Conseil de l'Ordre des avocats. Il joue un rôle déterminant dans l'obtention de la reconnaissance du statut professionnel de journaliste par la loi du 29 mars 1935 et se voit chargé d'en contrôler l'exercice par la déliurance de la carte professionnelle (33). Un mouvement similaire se fait jour chez les ingénieurs et techniciens : à l'Union syndicale des ingénieurs catholiques (USIC), qui existait déjà avant la guerre, s'ajoutent en 1919 l'Union des syndicats de techniciens et de l'industrie, du commerce et de l'agriculture (USTICA) et en 1920 l'Union des syndicats d'ingénieurs français (USIF), qui rassemble trois syndicats d'ingénieurs nés entre 1918 et 1919 (34). Le titre d'ingénieur est quant à lui réglementé par la loi du 10 juillet 1934.

\section{A la recherche d'une troisième voie entre étatisme et libéralisme}

Contrairement à ce qu' ont avancé certains sociologues et historiens anglo-saxons, on ne peut ni associer le mouvement de professionnalisation au libéralisme ni établir

(31) Cf. L. KARPIK, Les Avocats, op. cit., p. 170 ; P. PLAS, "La professionnalisation des avocats au début des années vingt. Enjeux, ruptures et nouveaux modèles ", in G. LE BÉGUEC (dir.), Avocats et barreaux en France 1910-1930, Nancy, Presses universitaires de Nancy, 1994, p. 59-76 ; C. Flllon, Histoire du barreau de Lyon sous la Troisième République, Lyon, Éditions Aléas, 1995.

(32) Cf. F. Muel-Dreyfus, Vichy et l'éternel féminin. Contribution à une sociologie politique de l'ordre des corps, Paris, Seuil, 1996, p. 301 et suiv. et M. DiNH, Les Médecins à la recherche d'un ordre 1845-1940, mémoire de maitrise, sous la direction de C. Charle, Université de Paris I, 1995.

(33) Cf. C. DeLPORTE, "Les journalistes dans l'entre-deux-guerres. Une identité en crise", Vingtième siècle, juillet-septembre 1995, p. 158-175 et C. CHARLE, Le Siècle de la presse (1830-1939), Paris, Le Seuil, 2004, chap. 12.

(34) Cf. A. Grelon (dir.), Les Ingénieurs de la crise, op. cit. et M. Descostes et J.-L. RoBerT (dir.), Clefs pour une histoire du syndicalisme cadre, Paris, Éditions Ouvrières, 1984. 
un lien de nécessité entre professionnalisation et autonomisation. Des travaux historiques, en particulier ceux de Konrad Jarausch, ont montré au contraire que des régimes autoritaires comme le Troisième Reich avaient pu satisfaire les revendications corporatistes de certaines professions, même si, à terme, l'instrumentalisation des experts pour les besoins de la machine de guerre nazie et leur implication dans l'entreprise d'extermination massive ont contribue à leur déprofessionnalisation (35). En Union soviétique, l'étatisation des activités intellectuelles et leur centralisation en un organisme unique, "l'union ", tout en améliorant nettement leurs conditions d'exercice, étaient un moyen de contrôle et de subordination de ces activités à des fins de propagande. On ne peut pas non plus assimiler un type d'organisation à un régime: on trouve en effet des ordres tels que celui des médecins en régime libéral, de même que l'organisation corporative du fascisme italien était fondée sur des syndicats. En revanche, le degré de fermeture du corps professionnel, la plus ou moins grande centralisation des différentes fonctions (solidarité, discipline, éthique, etc.) par un organe, le degré d'autonomie des instances par rapport à l'État du point de vue de la représentation du groupe professionnel (élus ou nommés), de l'élaboration d'une déontologie, de la police intérieure, la liberté d'adhésion aux instances représentatives, constituent des indicateurs de l'autoritarisme ou du libéralisme du régime.

Partout en Europe, le mouvement de professionnalisation et de fermeture des marchés s'accompagna d'une nationalisation des professions. Mais cette nationalisation prit des formes différentes selon les régimes: alors que dans le système de reproduction à composante scolaire mis en place par les régimes libéraux d'Europe occidentale la concurrence était censée opérer sur la base de la compétence, même si en réalité elle était généralement suspendue à la condition de nationalité et tendait à favoriser la reproduction sociale, dans les régimes autoritaires, elle fut limitée sur le fondement de la discrimination raciste, comme l'attestent les mesures d'exclusion des juifs de certaines professions libérales, qu'avait précédées l'instauration de numerus clausus à l'Université dans nombre de pays d'Europe centrale. En France, trois lois votées entre 1933 et 1935 avec le soutien des associations professionnelles restreignirent ainsi les possibilités d'exercer la profession médicale et celle d'avocat pour les étrangers et les "naturalisés ", un délai de dix ans étant imposé à ces derniers (36). S'inspirant en partie des modèles corporatistes fascistes (allemand, italien et portugais), le régime de Vichy allait adopter des mesures discriminatoires contre les Juifs et les enfants d'étrangers pour l'accès à la fonction publique et l'exercice des professions libérales. Les femmes furent aussi victimes des mesures de limitation de la concurrence dans les régimes fascistes et sous le régime de Vichy. Le processus de féminisation de ces professions et les résistances qu'il continue de susciter constituent de manière plus générale une constante de leur histoire.

(35) K. H. JaRausch, The Unfree Professions. German Lawyers, Teachers and Engineers, 1900-1950, New York, Oxford University Press, 1990. Voir aussi, sur les cas italien et hongrois, G. TuRl (ed.), Libere professioni e fascismo, Milan, F. Angeli, 1994, et M. KovaCs, Liberal professions and illiberal politics : Hungary from the Habsburgs to the Holocaust, Washington, D.C.-New York, Woodrow Wilson Center Press-Oxford University Press, 1994.

(36) G. NoIRIEL, Les Origines républicaines du régime de Vichy, Paris, Hachette, 1999, p. 141-149. 
La question de l'organisation professionnelle était débattue en France depuis la fin du XIXe siècle dans le cadre de la recherche d'une troisième voie entre capitalisme et socialisme, entre libéralisme et étatisme. Elle était préconisée, sous des formes qui pouvaient varier mais qui prenaient toutes la profession comme unité sociale et juridique pertinente, par les formations qui rejetaient aussi bien le capitalisme ultra-libéral que le syndicalisme révolutionnaire et la collectivisation des moyens de production et des services. De droite à gauche, toute une gamme de solutions fut proposée. Parmi celles-ci, il faut mentionner tout particulièrement, en raison du rôle structurant qu'ils eurent dans ces débats, les catholiques sociaux d'un côté, les réformistes de la gauche radicale-socialiste de l'autre.

Les catholiques sociaux promouvaient une organisation corporative de la société adossée à l'État, conformément à la doctrine sociale de l'Église promulguée par l'Encyclique Rerum novarum. Cette Encyclique donnait raison à René de La Tour du Pin contre les catholiques libéraux en légitimant l'existence de syndicats d'employeurs et d'employés séparés ainsi que l'intervention de l'État comme autorité d'arbitrage (37). Cette conception corporatiste explique la précocité des initiatives catholiques en vue de l'organisation des professions intellectuelles (dans la presse et chez les ingénieurs notamment), ainsi que les riches débats qui lui sont consacrés, l'accent y étant mis sur leur dimension spirituelle (38).

La conception de la gauche réformiste, soucieuse de réguler les relations professionnelles, peut être illustrée par le condensé théorique qu'en a donné Émile Durkheim dans la préface à la deuxième édition (1902) de son livre De la division du travail social (39). Il y développe l'idée que les groupements professionnels devraient, sous forme de corporations modernes, jouer un rôle de régulation de la vie économique qu'ils contribueraient à moraliser en assumant en outre - comme le font déjà certains syndicats - les fonctions de secours mutuel, d'œuvre éducative et d'organisation de la vie culturelle (40). Durkheim estime cependant nécessaire, du fait de leurs intérêts antagoniques, de maintenir, au sein d'une même branche professionnelle, la séparation de base entre syndicats d'employeurs et d'employés. La conception réformiste se retrouve en grande partie dans la tentative d'organisation des professions intellectuelles par la Confédération des travailleurs intellectuels fondée au lendemain de la Première Guerre mondiale, qu'aborde ici Alain Chatriot. Elle trouvera sa traduction dans la politique culturelle du Front populaire (41) et notamment dans le projet de loi sur le droit d'auteur et le contrat d'édition, évoqué dans l'article de Gisèle Sapiro et Boris Gobille.

(37) Cf. G. Cholvy et Y.-M. HiLAiRE, Histoire religieuse de la France contemporaine 1880-1930, Toulouse, Privat, 1986, p. 78-79.

(38) Voir H. SERRY, Naissance de l'intellectuel catholique, Paris, La Découverte, 2002, p. 223-249.

(39) E. DuRKheIm, De la division du travail social [1902], Paris, PUF, 1930, rééd. 1996, p. I-XLIV.

(40) Corps intermédiaires entre l'État et les individus, les corporations pourraient aussi, selon lui, devenir l'unité politique fondamentale, se substituant à la division territoriale qui fonde le système représentatif républicain.

(41) P. ORY, La Belle Illusion. Culture et politique sous le signe du Front populaire 1935-1938, Paris, Plon, 1994. 
Dans les années 1930, l'idée d'une organisation des professions était devenue prédominante (42). Alors que se tenait à Rome en 1935 le congrès sur les corporations, de nombreux projets visaient à instaurer une organisation corporative en France (43). Des juristes comme Georges Ripert tentaient parallèlement de faire de la profession un des facteurs de la condition juridique des individus (44). Le mouvement de professionnalisation culmina, en France, sous le régime de Vichy qui le détourna au profit d'un projet corporatiste. Mais le corporatisme préconisé par les traditionalistes qui tenaient les rênes du premier gouvernement se heurta très vite à l'étatisme du régime. Il allait bientôt "tourne[r] à la planification et au dirigisme", selon les termes de Robert Paxton (45). La loi du 16 août 1940 sur le contingentement des produits industriels prévoyait la mise en place de comités d'organisation par branche d'activité, dans une perspective strictement économique (46), tandis qu'une Charte du travail, à vocation plus sociale, devait préparer l'organisation des professions en corporations afin d'abolir la lutte des classes (47), à l'image de la corporation paysanne, constituée le 2 décembre 1940. En ce qui concerne les professions libérales, le gouvernement opta pour le modèle de l'ordre professionnel. Institués dans un esprit anti-syndicaliste, les ordres nouveaux reçurent le produit de la liquidation des biens des syndicats. Ainsi furent constitués dès 1940 les ordres des médecins et des architectes. D'autres allaient suivre, notamment ceux des dentistes, des sages-femmes, des vétérinaires, des experts-comptables, des géomètres-experts et de la police privée. Dans trois professions intellectuelles : les journalistes, les artistes et les écrivains, les projets d'ordres se heurtèrent néanmoins à la fois aux intérêts économiques des entreprises et à l'individualisme de leurs membres (48). Il semble

(42) Voir G. PIROU, Le Corporatisme. Corporatisme et libéralisme. Corporatisme et étatisme. Corporatisme et syndicalisme, Paris, Librairie du Recueil Sirey, 1935. Sur les enjeux politiques, économiques et intellectuels des débats autour de cette question, voir notamment A. CHATRIOT, La Démocratie sociale à la française. L'expérience du Conseil national économique, 1924-1940, Paris, La Découverte, 2002, et F. DENORD, Genèse et institutionnalisation du néo-libéralisme en France (1930-1950), thèse de doctorat de sociologie, EHESS, 2003.

(43) Deux propositions de loi d'ensemble furent déposées, l'une, en 1937, concernant l'organisation de la vie économique du pays, l'autre, en 1938, visant à instaurer une organisation corporative. Cf. G. VIANCE, Démocratie, dictature et corporatisme, Paris, Flammarion, 1938.

(44) G. RIPERT, Le Régime démocratique et le droit civil moderne, Paris, LGDJ, 1936.

(45) R. O. PAXTON, La France de Vichy 1940-1944, Paris, Seuil, 1973, p. 213. Voir aussi J.-P. LE CROM, "La politique sociale de Vichy : corporatisme ou dirigisme? ", in S. KAPLAN et P. MinARD (dir.), La France malade du corporatisme?, Paris, Belin, 2004, p. 403-425.

(46) H. Joly (dir.), Les Comités d'organisation et l'économie dirigée du régime de Vichy, Caen, Centre de recherche d'histoire quantitative, 2004.

(47) Cf. J.-P. LE CROM, Syndicats, nous voilà. Vichy et le corporatisme, Paris, Éditions de l'Atelier, 1995. Sur la doctrine corporatiste de Vichy, voir S. KAPLAN, "Un laboratoire de la doctrine corporatiste sous le régime de Vichy: l'Institut d'études corporatives et sociales ", Le Mouvement Social, nº 195, avril-juin 2001, p. 35-77.

(48) Cf. C. Delporte, Les Journalistes en France..., op. cit., chap. 10 ; L. BerTRAND-DorléAC, "L'Ordre des artistes et l'utopie corporatiste : les tentatives de régir la scène artistique française, juin 1940-août 1944 ", Revue d'Histoire moderne et contemporaine, janvier-mars 1990, p. 64-88 ; et Id., L'Art de la défaite 1940-1944, Paris, Seuil, 1993, p. 155-168. G. SAPIRO, "Entre individualisme et corporatisme: les écrivains dans la première moitié du XXe siècle", in S. KAPLAN et P. MINARD (dir.), La France malade du corporatisme?, op. cit., p. 279-314. 
qu'un projet de corporation regroupant les quatre branches des professions libérales, sanitaires, judiciaires, techniques et artistiques, qui rappelle la confédération italienne des professions artistiques et libérales sans qu'on puisse dire s'il en est directement inspiré, ait été envisagé début 1942, peu après la promulgation de la loi sur la Charte du travail en octobre 1941, mais il fut vite écarté (49).

L'exemple de l'Ordre des médecins montre bien les ambiguités du régime qui, tout en donnant satisfaction aux revendications corporatistes, les a inscrites dans un cadre étatiste contraire au projet des traditionalistes. Ainsi la création de l'Ordre répondait au vœu prononcé depuis 1929 par la Confédération des syndicats médicaux, pour mieux contrôler les conditions d'exercice de la profession : remplaçant la Confédération dissoute, l'Ordre, auquel l'affiliation est obligatoire, est ainsi chargé de l'application des nouvelles lois excluant les étrangers même naturalisés et imposant aux Juifs un numerus clausus. Mais cet Ordre aux pouvoirs centralisateurs, dont les membres sont nommés par le gouvernement, heurte la tradition libérale prédominante dans la profession, à tel point qu'il sera réorganisé par la loi du 10 septembre 1942 dans un sens plus libéral (séparation des pouvoirs disciplinaires et professionnels, élections partielles des représentants aux conseils départementaux et au conseil national) (50).

Le mouvement de professionnalisation n'a pas pris fin immédiatement avec le régime de Vichy. L'épuration professionnelle, qui s'opère sur le modèle des administrations et par application de l'ordonnance sur l'épuration administrative, s'inscrit très directement dans ce mouvement (51). Les ordres créés sous Vichy ainsi que les mesures prises contre les syndicats sont toutefois annulés. Refondés, les nouveaux ordres (des médecins, des architectes, des experts-comptables) ne disposent plus des pouvoirs réglementaires qui leur avaient été octroyés en 1940 mais uniquement d'un rôle consultatif dans l'élaboration des règles professionnelles.

La tendance dite "néo-corporatiste ", qui mettait l'accent sur l'organisation professionnelle, n'allait pas tarder à être renversée à la faveur de la diffusion internationale de la doctrine néo-libérale (52) et de la pression américaine pour l'ouverture des marchés (53). Ce mouvement n'empêcha pas que des revendications professionnelles anciennes ou élaborées dans le cadre de la Résistance aboutissent sous les IV et Vépubliques, à la faveur du dirigisme planificateur : réorganisation de la profession de journaliste à la Libération, création d'une Caisse des lettres en 1946 et loi sur le droit d'auteur en 1957, création de l'École nationale de la magistrature et

(49) Ce projet est évoqué lors d'une assemblée de la Société des gens de lettres (voir infra) ; M. N[Ö̈L], "La lutte de la Société des Gens de Lettres contre les épreuves du temps ", Le Figaro, 21-22 mars 1942.

(50) Cf. F. Muel-DreYFus, Vichy et l'éternel féminin, op. cit., p. 301-311.

(51) Voir en particulier M. O. BARUCH (dir.), Une poignée de misérables. L'épuration de la société française après la Seconde Guerre mondiale, Paris, Fayard, 2003. Développée sous l'Occupation allemande, l'idée de fonder un droit professionnel autonome survit aussi quelque temps au régime de Vichy. Voir J. SAVATIER, Étude juridique..., op. cit.

(52) F. DENORD, "Le prophète, le pèlerin et le missionnaire. La circulation internationale du néolibéralisme et ses acteurs ", Actes de la recherche en sciences sociales, n 145, décembre 2002, p. 9-20.

(53) Rappelons que, pour la France, les accords Blum-Byrnes conclus en 1946 prévoient l'ouverture du marché français aux produits américains dans tous les secteurs, notamment le cinéma. 
réforme hospitalo-universitaire en 1958, etc. (54). La mobilisation de mai 1968 a renouvelé la réflexion des groupes professionnels sur leur organisation, comme en témoignent les revendications des auteurs et des responsables éditoriaux pour participer aux décisions au sein des maisons d'édition en Allemagne, permettant, ainsi qu'il arrive souvent dans les moments de crise, la cristallisation d'une nouvelle génération et la création de nouvelles instances, telle l'Union des écrivains en France (55). Ce moment a favorisé également l'aboutissement de revendications professionnelles anciennes : en 1969 et 1975 respectivement les artistes et les auteurs obtiennent le statut de salariés (56), en 1977 une loi sur l'architecture accorde le monopole de l'exercice aux architectes diplômés (en Allemagne, la Chambre des architectes fut créée en 1959).

Cependant, tandis que le mouvement de professionnalisation des activités intellectuelles culminait sous l'effet du dirigisme planificateur et que la rationalisation des moyens de production entraînait la bureaucratisation de nombre d'entre elles (hôpitaux, instituts de recherche, grands groupes de l'édition, multinationales des industries culturelles), un processus de libéralisation se profilait parallèlement, dès la signature des accords du GATT en 1948, sous l'impulsion des États-Unis. A partir de la fin des années 1980, l'extension des accords de libre-échange du GATT aux services dans le cycle de l'Uruguay a posé à ces professions intellectuelles le problème de la déréglementation. Au nom de la lutte contre les monopoles, celle-ci menace en effet la réglementation et les politiques d'aides mises en place pour soutenir les productions culturelles nationales dans le domaine de l'audiovisuel (quotas de diffusion), du cinéma (aides) et du livre (prix unique, protection du droit moral de l'auteur). Reste à savoir dans quelle mesure cette déréglementation s'accompagnera ou non d'une déprofessionnalisation ou d'une recomposition de la division du travail intellectuel et d'expertise.

(54) A. BOIGEOL, "La formation des magistrats : de l'apprentissage sur le tas à l'école professionnelle ", Actes de la recherche en sciences sociales, n 76-77, mars 1989, p. 49-64; A. SIMONIN, "Le Comité médical de la Résistance : un succès différé ", Le Mouvement Social, n 180, juillet-septembre 1997, p. $159-178$.

(55) Voir B. GoBILLE, Crise politique et incertitude: régimes de problématisation et logiques de mobilisation des écrivains en mai 68, thèse de doctorat, sous la direction de B. Pudal, EHESS, 2003 ; J.-L. VIoLEAU, Les Architectes et mai 68, Paris, Éditions Recherches, 2005.

(56) Sur les paradoxes de cette "reprofessionnalisation " des artistes, voir R. MouLIN, " De l'artisan au professionnel : l'artiste", Sociologie du travail, n 4, octobre-décembre 1983, p. 388-403. 